\title{
PROSPECTIVA EN EL ENTORNO EMPRESARIAL NACIONAL
}

\section{PROSPECTIVE OF THE NATIONAL BUSINESS ENVIRONMENT}

\section{Adrián Alejandro Flores Konja*}

Docente Principal de la Facultad de Ciencias Contables, UNMSM

\section{Manuel Alberto Hidalgo Tupia**}

Docente de la Facultad de Ciencias Contables, UNMSM

[Recepción: Setiembre de 2010 / Conformidad: Setiembre de 2010]

\section{RESUMEN}

La prospectiva ha irrumpido con fuerza en la planificación de empresas e industrias, promovida muchas veces por los gobiernos nacionales. Se la concibe como un enfoque de un proceso de planificación consistente en identificar y lograr progresivamente las condiciones para el éxito futuro, entendido como el logro de la visión de la organización. En el presente artículo se reseña los conceptos básicos de la prospectiva y los enfoques y herramientas de su aplicación.

El presente artículo es fruto de una investigación exploratoria, básicamente bibliográfica, cuyo objetivo es explorar la adecuación de los conceptos de prospectiva con las características del entorno empresarial peruano, a fin de optimizar la aplicación del concepto y metodología prospectiva a nuestro entorno. Como explicaremos a continuación, esta relación de entorno empresarial y planificación, en el contexto de un Plan Industrial Nacional y las exigencias de competitividad del entorno global es más ventajosamente entendida desde el enfoque de la prospectiva.

Palabras clave: Prospectiva, Empresa, Entorno empresarial, Planeación, Estrategia, Futuro, Futurología, Predicción, Tendencias, Competitividad, Plan industrial, Industrialización, Cadenas productivas, Estado, Nación.

\begin{abstract}
Foresight has burst in the planning of business and industry, often promoted by national governments. Is conceived as an approach to a planning process involved in identifying and achieving progressively the conditions for future success, defined as the achievement of the vision of the organization. This article outlines the basics of Foresight and approaches and tools for its implementation.

This article is the result of exploratory research, basically literature, whose aim is to explore the adequacy of the concepts of foresight with the characteristics of Peruvian business environment in order to optimize the application of the concept and methodology prospectively to our environment. As explained below, this relationship of the business environment and planning in the context of a National Industrial Plan and the requirements of a competitive global environment is understood more advantageously from the viewpoint of foresight.
\end{abstract}

Key words: Foresight. Company. Business environment. Planning. Strategy. Future. Futurology. Prediction. Trends. Competitiveness. Industrial Plan, Industrialization. Chain. State. Nation.

\footnotetext{
* Doctor en Ciencias Contables y Empresariales - UNMSM, Contador Público Colegiado Certificado, actual Decano de la Facultad de Ciencias Contables, UNMSM. E-mail: afloreskonja@yahoo.es

** Economista, UNMSM. Docente de la Facultad de Ciencias Contables, UNMSM. E-mail: mhidalgot@hotmail.com
} 


\section{INTRODUCCIÓN}

La prospectiva es un concepto y una metodología que ha irrumpido con fuerza en la planificación de organizaciones, es decir, empresas, industrias y aun de territorios en los últimos años. Se la concibe como en enfoque de un proceso de planificación consistente en identificar y lograr progresivamente las condiciones futuras para el éxito, sea este el logro del objetivo o de la Visión de la organización. El término viene del latín prospicere, mirar a lo lejos o mirar desde lejos, y fue usado en su sentido actual por primera vez por el francés Gastón Berger, en 1957, como algo distinto a la previsión y su correlato, la predicción.

Prospectiva no es fundamentalmente previsión y predicción, sino el ir moldeando desde el presente las condiciones futuras, en una actitud esencialmente proactiva. Es decir, la Prospectiva no consiste básicamente en conocer las condiciones futuras, o, lo que es lo mismo, conocer los cambios que se están produciendo o podrían producirse, sinó en la construcción de estas condiciones futuras desde el presente, conforme a los propósitos o visión de la organización.

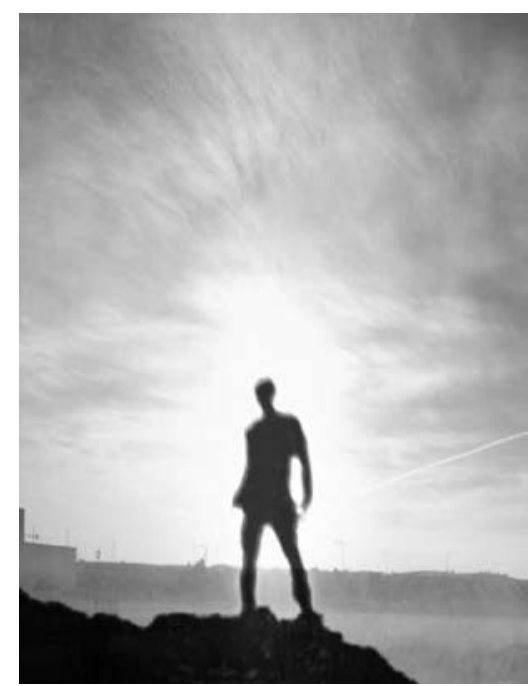

Prospectiva: Parte de "mirar desde lejos, mirar a lo lejos"

\section{PROSPECTIVA. DEFINICIÓN Y OBJETIVOS}

No existe una definición formal aceptada universalmente de prospectiva; ni tampoco una que distinga la prospectiva de otras actividades orientadas a estudios de futuro. Sin embargo, la diversidad de definiciones existentes, presentan un concepto unificador.

- Gastón Berger la define como la "Ciencia que estudia el futuro para comprenderlo y poder influir en él”.

- Real Academia de la Lengua Española la define como: "Conjunto de análisis y estudios realizados con el fin de explorar o de predecir el futuro en una determinada materia".

- La OCDEla plantea así: "Conjunto de tentativas sistemáticas para observar a largo plazo el futuro de la ciencia, la tecnología, la economía y la sociedad con el propósito de identificar las tecnologías emergentes que probablemente produzca los mayores beneficios económicos y/o sociales."

- Van de Wilt y Hans Rutten Putch, la definen como: "Un proceso sistemático interactivo que explora dinámicas futuras en ciencia, tecnología, la economía y la sociedad, afín de identificar estrategias y acciones viables para los stakeholders."

La prospectiva tiene un soporte filosófico establecido por la Escuela Francesa, cuyos exponentes más reconocidos han sido Bernard y Hugues de Jouvenal y Michel Godet. Sus principales proponentes y auspiciadores en la actualidad son el Laboratorio de Investigación en Prospectiva Estratégica y Organización, LIPSOR, del Conservatorio Nacional de Artes y Oficios (CNAM), y el Instituto Europeo de Prospectiva y Estrategia. Godet es profesor titular de la Cátedra de Prospectiva Industrial del CNAM, y dirige también LIPSOR. Además de su concepto, 
la prospectiva se ha conocido como el método o la técnica de su aplicación a una organización, como veremos, según un grupo de técnicas elaboradas por diferentes expertos y propuestas también por LIPSOR y CNAM.

Esquemáticamente la prospectiva se fundamenta en 3 momentos -triángulo griegoque son la anticipación, la apropiación y la acción. Los tres componentes del triángulo griego son: "Logos" (el pensamiento, la racionalidad, el discurso), "Epithumia" (el deseo en todos sus aspectos nobles y menos nobles), "Erga" (las acciones, las realizaciones), (Godet, 2007, p.18). La anticipación como el pensamiento colectivo que ve hacía el futuro; la acción como deseo estratégico y la apropiación como movilización colectiva.

Como dice Godet, el azul de la razón fría asociado al amarillo de las sensaciones calientes produce el verde de la acción brillante (Godet, 2007: 18)

Gráfico N.o 1. El triángulo griego.

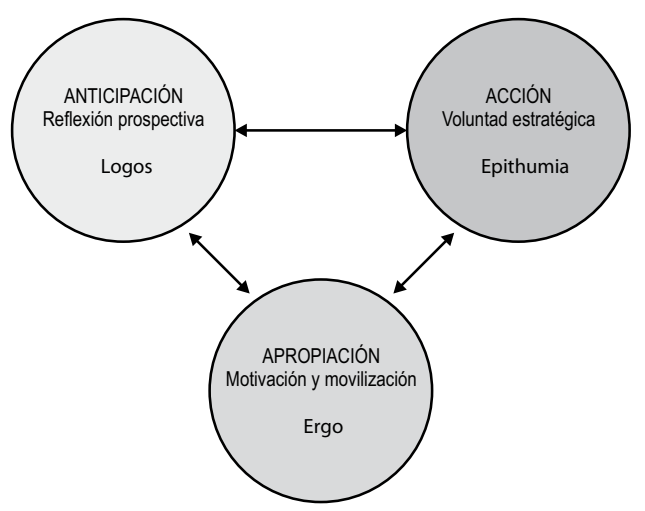

Los objetivos de la prospectiva (Indacochea, 2010) son:

- Señalar la dirección de los futuros posibles, distinguiendo dentro de hechos del presente, aquellos portadores del futuro.

- Anunciar la ocurrencia de un evento futuro, para propiciarla o para impedirla.

- Orientar la acción presente a la luz de futuros deseables.
- Complementar a la Planeación tradicional con una visión más amplia y a más largo plazo.

- Adoptar una visión global y sistémica en el objeto estudiado.

\section{PROSPECTIVA, PRONÓSTICO Y DETERMINISMO: DIFERENCIAS Y SIMILITUDES}

En opinión de Hugues de Jouvenel y Pierre Masse, citados por Godet, el acuñamiento del término prospectiva "nació de una rebelión del espíritu contra el yugo del determinismo y el juego de azar". Es decir, la prospectiva considera que el futuro no está ya predeterminado por una Ley de Hierro de la necesidad; tampoco el futuro descansa en la incertidumbre total, como si Dios jugase a los dados; por el contrario, la voluntad activa del individuo y las comunidades también influye grandemente en delinear el futuro.

De hecho, la prospectiva es un enfoque diferenciado fundamentalmente con la futurología, dado que, como dijimos, no consiste básicamente en conocer las tendencias que modelarán el futuro, o anticiparse a los cambios, sino que implican una voluntad de moldear el futuro desde el presente, $\mathrm{o}$, como se ha dicho también, "moldear el presente desde el futuro".

Una formulación que une estos elementos la dan Acuña y Konow (1990. 6, citados por Escobar), donde expresan el futuro deseado, simbolizado por $\mathbf{F}$, combinando 3 factores: 1) tendencia o inercia histórica, simbolizada por $\mathbf{T} ; 2)$ Evento o acontecimiento inesperado, E, y 3) propósitos u objetivos individuales (P).

La "fórmula" que expresa la relación sería:

$$
\mathrm{F}=\mathrm{a} \mathrm{T}+\mathrm{b} \mathrm{E}+\mathrm{c} \mathrm{P}
$$

Siendo a, b y c parámetros de valor positivo, los cuales, para conservar la lógica, deben sumar 1 . 
Una perspectiva determinista haría que todo dependiese de $\mathbf{T}$, siendo $\mathrm{a}=1, \mathrm{y}$ b y $\mathrm{c}=$ 0 ; es decir, ni los eventos azarosos ni los propósitos humanos tendrían peso alguno en delinear el futuro. Una perspectiva del azar haría que todo dependiese de $\mathbf{E}$, siendo los parámetros a y c $=0$. Lo propio de la prospectiva es la inclusión de la variable $\mathbf{P}$, que influye preponderantemente en el análisis y en la determinación de $\mathbf{F}$.

$\mathrm{Al}$ respecto, sostiene Escobar que "el deseo que algo ocurra influye en que eso sea así y las imágenes o deseos de la gente influyen en el futuro. Esa parte es elegible, ya que depende de la voluntad de las personas y además es diseñable. De este modo estamos creando un futuro gracias a nuestra imaginación”. Es decir, el deseo eficiente de que algo ocurra es un factor muy importante para que realmente ocurra.

Analicemos en siguiente gráfico para mayor ilustración.

Gráfico N.o 2. Enfoque de la prospectiva.

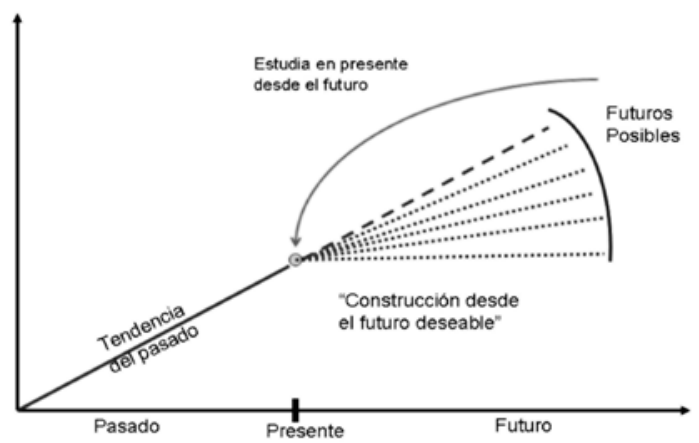

Fuente: Adaptado de Miklos, Tomas, ¿Cómo construir el futuro? Acercamiento a la Prospectiva. Estudios Prospectivos. México, p. 4. Citado por Indacochea.

Representamos tres tiempos, pasado, presente y futuro. El pasado es la fuente de las tendencias que van hasta el presente y se prolongan hacia el futuro, como una fuerza potencial. Sin embargo, estas tendencias no van a ser totalmente determinantes, sino que podemos concebir los "futuros posibles", distintos y alternativos, y de entre ellos podemos elegir un "futuro deseable", y a partir de esta elección, definimos las condiciones presentes necesarias para ese "futuro deseable", y planificamos su logro, por eso se habla de la "construcción desde el futuro deseable". Por ello decimos que la prospectiva "estudia el presente desde el futuro".

\section{PROSPECTIVA, FUTURO E INCERTIDUMBRE}

Un deseo humano presente siempre, pese a su imposibilidad, ha sido el de conocer los sucesos futuros; especialmente, debido a que su desconocimiento produce incertidumbre, un estado cognitivo y anímico poco deseable. Este deseo ha sido sentido desde siempre por la Humanidad, enfocándose por distintos medios, irracionales o racionales, por ejemplo, por la adivinación, en el primer caso, el pronóstico y la proyección, en el segundo caso, y en diversas modalidades. Si hablamos de los medios racionales, entendemos que, aunque no pueden desaparecer la incertidumbre en la persona o agente de decisión, puede reducirla, al medir la posibilidad de que ocurra un hecho, o un conjunto de hechos, sean deseables o no.

La incertidumbre puede tener diversas intensidades, (Cornet, 2001, citado por Indacochea), desde

1. Una incertidumbre irrelevante, o Futuro Claro, en la cual hay un solo futuro posible.

2. Poca incertidumbre, donde existe un número conocido y limitado de futuros posibles (Futuros alternativos).

3. Alta incertidumbre, donde los futuros posibles son muchos (Abanico de futuros).

4. Incertidumbre total, donde se desconoce los futuros posibles, o Ambigüedad verdadera. 
El mayor reto de la aplicación de la prospectiva es tener en claro y definir el nivel de incertidumbre relativo a las decisiones a tomar sobre el futuro, que están bajo consideración. Si no hay incertidumbre (incertidumbre residual cero), no hay necesidad de la prospectiva y un simple pronóstico es suficiente para tomar decisiones. Se ha sostenido que la prospectiva busca pasar de los niveles 3 y 4 a los niveles 1 y 2 .

\section{PROSPECTIVA, PLANIFICACIÓN Y ESTRATEGIA}

Aunque los tres conceptos comparten muchos elementos, y concurren en un único propósito, especialmente cuando se aplican a las empresas, sus diferencias no pueden pasarse por alto. En especial, algunos autores utilizan los términos en forma intercambiable, como si fueran sinónimos, lo que lleva a confusiones $\mathrm{y}$, consiguientemente, a malas aplicaciones.

Existe una polisemia del término 'estratégico', que puede significar cosas diferentes, desde, "importante" o "irreversible", hasta "lo opuesto a lo táctico". Clausewitz (Op. Cit., p 76) decía que lo táctico es la conducción de la fuerza para decidir el resultado de un encuentro individual (batalla), mientras que estrategia es la combinación de los encuentros para decidir el resultado de la guerra. La prospectiva es en esencia estratégica, por el hecho de "mirar a lo lejos, o desde lejos", de hecho, por ello se habla mucho de prospectiva estratégica, así como de "planificación estratégica" y "gestión estratégica”. Por su parte, la prospectiva es obviamente planificación, aunque no es únicamente planificación, visto como parte del proceso administrativo clásico de Planificación, organización, dirección $y$ control, sino que es un proceso que intervendrá en las demás fases.
Pero, resulta claro que no toda planificación es prospectivista, ni todo proceso estratégico es prospectivista, pues la prospectiva es un concepto más particular, con características propias en concepto y en metodología.

Sostiene Godet (2007: 9) que "la definición de la planificación propuesta por Ackoff (1973), "Concebir un futuro deseado así como los medios necesarios para alcanzarlo", no difiere en absoluto de la definición que nosotros proponemos para la prospectiva, donde el sueño fecunda la realidad, donde el deseo y la intencionalidad es fuente productora de futuro, donde la anticipación ilumina la preactividad y la proactividad".

Desde fines de los años ochenta se difunde la expresión de prospectiva estratégica; pues, como dice Godet (2007: 10) "¿Cómo podríamos pensar en actuar como un estratega sin "mirar a lo lejos, a lo ancho, a lo profundo; tomar riesgos, pensar en el hombre" tal como lo hace la prospectiva y así lo comenta Gaston Berger (1954). ¡Por supuesto que sería imposible! Y, en sentido inverso, para Gaston Berger "contemplando el futuro se transforma el presente"; de este modo la anticipación invita a la acción". "Para nosotros, -agrega Godet (loc. cit)-, "el asunto es evidente, la prospectiva resulta muy a menudo estratégica caso de no serlo por los avances que provoca, sí por la intención que lleva y la estrategia se vuelve necesariamente prospectiva si desea iluminar las opciones que comprometen el futuro".

Puntualizando sobre la relación entre prospectiva y estrategia, nos referimos a las reflexiones de Godet, que señala los aspectos de exploración y preparación para la acción que vemos en la prospectiva. "Esta dicotomía entre la exploración y la preparación de la acción” -señala Godet (2007: 13-14)- nos lleva a distinguir cuatro cuestiones funda- 
mentales: ¿Qué puede ocurrir? (Q1), ¿Qué puedo hacer? (Q2), ¿Qué voy a hacer? (Q3), ¿Cómo voy a hacerlo? (Q4). Esta cuestión previa sobre la identidad de la empresa, frecuentemente olvidada, es el punto de partida para la metodología estratégica de Marc Giget (1998)". Añade que así "se impone una vuelta a las fuentes sobre las raíces de las competencias, sus fortalezas y debilidades, recordando la famosa frase "conócete a ti mismo".

¿Cuál es la relación entre prospectiva y estrategia, vista desde esta perspectiva? "La prospectiva, cuando va sola - dice Godet (opcit.)-, se centra sobre (Q1) ¿Qué puede ocurrir? Se convierte en estratégica cuando una organización se interroga sobre el ¿Qué puedo yo hacer? (Q2). Una vez ambas cuestiones hayan sido tratadas, la estrategia parte del ¿Qué puedo yo hacer? (Q2) para plantearse las otras dos cuestiones: ¿Qué voy a hacer yo? (Q3) y ¿Cómo voy a hacerlo? (Q4)”. De ahí se deduce la conexión que existe entre la prospectiva y la estrategia.

Cuadro No 1. Prospectiva y estrategia

\begin{tabular}{|c|l|}
\hline PREGUNTAS & \multicolumn{1}{|c|}{ ENFOQUE } \\
\hline (Q1) ¿Qué puede ocurrir? & Prospectiva sola \\
\hline (Q2) ¿Qué puedo yo hacer? & Estrategia \\
\hline (Q3) ¿Qué voy a hacer yo? & Formulación estratégica \\
\hline (Q4) ¿Cómo voy a hacerlo? & Formulación estratégica \\
\hline
\end{tabular}

\section{ENFOQUES Y HERRAMIENTAS DE LA TÉCNICA PROSPECTIVISTA}

La prospectiva se conoce quizás, tanto por su concepto, por los elementos de su técnica de aplicación; algunas de las herramientas usadas en la prospectiva son:

- Análisis de megatendencias

- Análisis retrospectivo
- Análisis estructural

- Entrevista a profundidad

- Método Delphi

- Ábaco de Regnier

- Árboles de relevancia

- Análisis morfológico

- Escenarios

- Extrapolación de tendencias

- Matrices de impacto cruzado

- Matrices de selección

- Análisis de dinámica de sistemas.

En muchos casos, se le da más importancia al empleo masivo de técnicas como el Ábaco de Regnier, el método Delphi, el Análisis Estructural, la Matriz de Impacto Cruzado, los Escenarios, el Árbol de Pertinencia y el Grupo Vigía, etc., que el factor de la coherencia de conjunto y la participación de los actores como los verdaderos agentes del cambio. $\mathrm{Y}$ aunque cada una de las técnicas mencionadas son de una gran utilidad, incluso en forma individual, y aplicadas -aún fuera del concepto de prospectiva- en la gestión lisa y llana propiamente dicha, el concepto central y la coherencia de la aplicación es vital, por el valor propio del concepto de prospectiva. La prospectiva se conoce hoy como el empleo de todas o algunas de las técnicas denominándose como lo hace Godet, como el "método de escenarios".

Vale aclarar sobrelos escenarios. Aunquelos factores o variables pueden preverse o estimarse, sus relaciones también deben considerarse en su conjunto, y se hace a través del concepto de "escenarios", como las distintas combinaciones que se pueden establecer de acuerdo a los diversos valores atribuidos a las variables.

¿Podemos analizar todas las combinaciones o escenarios posibles? Es claro que no, pero no es necesario, si barremos las posibilidades más generales, dejando las posibilidades intermedias, tendremos un conocimiento suficiente de todas las posibilidades (escenarios). 
Nos ha tocado vivir en una época en la que los cambios se suceden a un ritmo nunca antes visto, y por ello, la labor de planeamiento se ve fuertemente impactada por los desarrollos que suelen tomar los acontecimientos sociales, económicos, culturales y políticos. Por esa razón, dice Ortega, "ya no es posible realizar el planeamiento estratégico clásico, ni en lo político ni en lo empresarial, basados en una "visión" única y siempre deseable para la organización, sino que es preciso contar con estrategias robustas y planes contingentes basados en diferentes escenarios posibles y probables. Es aquí donde la prospectiva produce su mayor beneficio."

Un segundo enfoque, aún más básico, considera que la guía básica de la prospectiva consiste en los siguientes pasos:

- Delimitación del problema

- Identificación de las tendencias

- Identificación de rupturas

- Identificación de variables estratégicas

- Evaluación del poder de los actores

- Construcción de escenarios

- Determinación de objetivos, estrategias, acciones

- Funciones de Vigía y control
Estos enfoques no son contrapuestos, porque las técnicas se relacionan a los pasos, aunque no se identifican exactamente.

Un tercer enfoque se centra en el tratamiento que se da a algunos de los sujetos que participan, concretamente, el facilitador, los actores, el grupo Vigía y los expertos, que, en conjunto con los demás son los agentes de cambio. Este enfoque pone el énfasis en la transformación del individuo, de las organizaciones, y en la forma de asignar los recursos para construir un futuro deseado.

Otros autores asimilan el método de la prospectiva al método científico, llanamente, enfocando la prospectiva como predicción, con los cambios respectivos. Es decir, para cumplir con las fases del método se aplica el método general científico, para lo cual, la metodología de la investigación aporta normas que no se pueden sustituir sin una razón sustentada que pueda presentarse para un caso particular específico.

En el siguiente gráfico se muestra en forma genérica el uso de las distintas técnicas de la prospectiva dentro de una metodología integrada, como lo propone Godet. 
Gráfico No 3. Planificación estratégica por escenarios: metodología integrada.

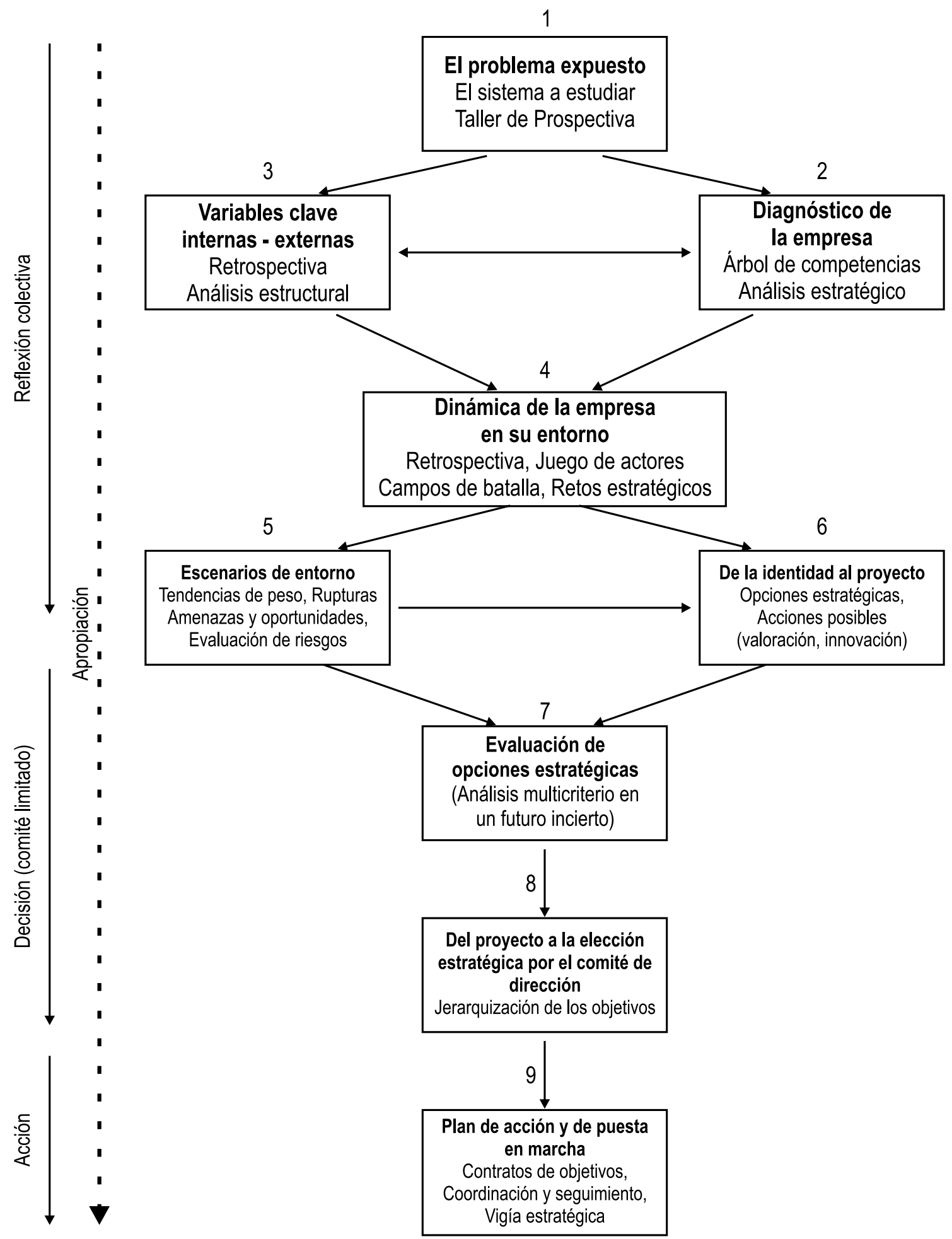

Fuente: Godet, 2007.

62/ QVIPURAMAYoc |Vol. 17(2) 2010 


\section{PROSPECTIVA EN EL ENTORNO EMPRESARIAL PERUANO}

La prospectiva es relativamente nueva en el Perú, establece Ortega San Martín, y su aplicación a las empresas peruanas también es incipiente; aún cuando partes de las técnicas prospectivistas se aplican en forma aislada, falta difundir en concepto básico de las prospectiva como el elemento clave. Como antecedentes, sabemos que el Consejo Nacional de Ciencia y Tecnología, CONCYTEC ha trabajado en difundir el concepto de prospectiva, habiéndose formado ya varios cientos de profesionales en prospectiva.

La aplicación de la prospectiva en nuestro país distará mucho de la aplicación en los países desarrollados, por ejemplo, como se ejemplifica en la aplicación en el contexto de Europa, por la especificidad del Perú y de la región, que muestran grandes diferencias. Nuestras empresas:

- Son consumidoras de tecnología, no productoras, mostrando nuestro país un gran retraso en Investigación y Desarrollo tecnológico;

- Son "tomadoras" de las condiciones del mercado global, sin un apoyo que les permita aprovechar sus oportunidades y remontar las amenazas del entorno global;

- No existe un plan Nacional Industrial, que es la voluntad expresa de la Sociedad, a través del Estado, de promover determinados sectores industriales logrando en ellas competencias de rango global;

- Si hablamos de empresas peruanas, apuntaremos a las que están internacionalizadas o en vías de internacionalizarse, para lo cual, deben tomar dos alternativas, según el sector industrial en que se encuentren: $\mathrm{o}$ aprovechan las ventajas competitivas ya existentes para nuestro país, o se enfocan en crear ventajas competitivas nuevas, en base a la innovación. Pero aquí surge la situación problemática: el Perú actual está atado a las ventajas "competitivas" dotadas por la su geología (una inmensa riqueza minera) y su clima, que nos convierte en un país exportador de bienes primarios extractivos. En cambio, si buscamos ampliar nuestras ventajas competitivas intencionalmente, a otras que nos permitan una mejor inserción en el comercio internacional; entonces necesitamos un Plan Industrial; en donde nos atreveremos a concebir una serie de sectores industriales adelantados que muchos no se atreverían siquiera a pensar, que el Perú en la actualidad "no está en condiciones" de adquirir. Este Plan puede ser preparado haciendo uso de los conceptos de la prospectiva. Recordemos que la prospectiva industrial es una de las principales aplicaciones de la prospectiva, más allá de su aplicación a empresas individuales, pero que nos permitiría tener empresas de rango mundial.

Algunos consideran que las ventajas competitivas sólo pueden provenir de las condiciones naturales, o ser determinadas por las tendencias o las condiciones actuales del comercio, la dotación de factores productivos o la acumulación de capital (que serían la " $\mathrm{T}$ " de la "fórmula" del futuro), que muestran a nuestro país como deficitario de capitales y tecnología. Si embargo, la evidencia mostrada por autores como Porter muestra que los países crean sus ventajas competitivas por encima, y aun, en sentido opuesto a las supuestas ventajas "naturales" o "históricas", como en el caso de los países recientemente industrializados, que acentuaron en los propósitos ("P" de la fórmula del Futuro).

En los países desarrollados, la Prospectiva industrial es iniciada por los gobiernos, como por ejemplo en Japón, con el NISTEP 
(Instituto Nacional de Planeamiento Científico y Tecnológico), Corea y en nuestro continente, Brasil, tendiendo a una alianza Estado "Industria" Universidad. Estos enfoques han promovido la formación de cadenas productivas, por ejemplo, en Brasil las cadenas priorizadas han sido construcción civil, textiles y confecciones, maderas y muebles y plásticos. De hecho en nuestro país, el ámbito natural para la prospectiva es esa alianza de Empresas - Estado y Universidades, más allá de ideologismos de cualquier signo que reemplacen la realidad de la necesidad imperiosa de una Prospectiva Industrial Nacional compartida como Proyecto por todos.

\section{CONCLUSIONES}

Como una conclusión de la investigación preliminar aquí reseñada, producto del análisis bibliográfico, podemos decir que:

- El enfoque prospectivo se está imponiendo cada vez con mayor intensidad en los procesos de planificación en empresas e industrias, constituyendo una fase más avanzada del concepto de planeación estratégica.

- Este enfoque puede también aplicarse al caso de la planeación del desarrollo de determinados sectores industriales de una nación.

- El enfoque prospectivo sería de gran ventaja frente a los anteriores enfoques, debido a que enfoca el tema de la "voluntad" sobre el determinismo u otros factores.

- El ámbito natural para la prospectiva es la alianza de Empresas - Estado y Universidades, lo que permitiría superar la ubicación desventajosa de las empresas peruanas ante un entorno global fuera de su control.

- Para una mejor aplicación de la prospectiva para el planeamiento de empresas e industrias debe participar fuertemente el Estado, debido a que poseer empresas de rango mundial no es posible sin el apoyo organizado de toda la nación.

\section{REFERENCIAS BIBLIOGRÁFICAS}

1. Cournet, Hugh. Foresight. Crafting Strategy in an Uncertain World. Harvard Business School Press. 2001. Citado por Indacochea, op. Cit.

2. Clausewitz, Karl von. De la Guerra. Instituto Cubano del Libro. Biblioteca del Oficial. La Habana, 1969, 716 p.

3. Godet, Michel, y Philippe Durance. La caja de herramientas de la prospectiva estratégica. Problema y Métodos. Cuaderno N.o 20. Prospektiker. Segunda edición, enero de 2007, 105 p.

4. Godet, Michel. De la anticipación a la acción. Bogotá: Editorial Alfaomega Marcombo, 1995, $360 \mathrm{p}$.

5. Indacochea Cáceda, Alejandro. PROSPECTIVA El nuevo enfoque para los negocios. En: CENTRUM CATÓLICA (www.centrum.pucp.edu.pe) Consultado el 23-04-10.

6. Miklos, Tomas, ¿Cómo construir el futuro? Acercamiento a la Prospectiva. Estudios Prospectivos. México. Citado por Indacochea.

7. Ministerio de Educación, Dirección de Coordinación Universitaria. La Universidad en el Perú. Informe 2006. Razones para una Reforma Universitaria. MINEDU. Julio 2006, 197 p.

8. Ortega San Martín, Fernando. La Prospectiva, herramienta indispensable de planeamiento en una era de cambios.

9. Restrepo, Francisco. Taller de Prospectiva y sus Aplicaciones a la Planeación Estratégica, Centro IESA del Zulía, Maracaibo. Citado por Indacochea. 